\title{
Active Control
}

National Cancer Institute

\section{Source}

National Cancer Institute. Active Control. NCI Thesaurus. Code C49649.

The use of a pharmacologic substance as a control within a treatment regimen where

the pharmacologic properties of that substance are relevant to the condition being treated. 